Recebido em 09/2013. Aceito para publicação em 03/2014.

\title{
REGISTRO DA OCORRÊNCIA DE ENCHENOPA BEEBEI (HAVILAND) (HEMIPTERA, MEMBRACIDAE) PARA O BRASIL
}

\section{REGISTRATION OF OCCURRENCE OF ENCHENOPA BEEBEI (HAVILAND) (HEMIPTERA, MEMBRACIDAE) FOR BRAZIL}

\section{Frederico Lencioni-Neto ${ }^{1}$ \\ Albino M. Sakakibara ${ }^{2}$}

Resumo: Este trabalho apresenta o primeiro registro de Enchenopa beebei (HAVILAND, 1925) para o Brasil. Dois exemplares (macho e fêmea) foram coletados em pequenas áreas remanescentes da Mata Atlântica, pertencentes à Universidade do Vale do Paraíba, localizadas, respectivamente, no Campus de Villa Branca (Jacareí) e de Urbanova (São José dos Campos), no Estado de São Paulo, Brasil. A espécie é redescrita e ilustrada.

Palavras-chave: distribuição geográfica; Mata Atlântica; Membracinae; taxonomia.

\begin{abstract}
Occurrence record of Enchenopa beebei (Haviland) (Hemiptera, Membracidae) in Brazil. In this paper, Enchenopa beebei (Haviland, 1925) is recorded for the first time in Brazil. Two specimens (male and female) were collected in small remaining areas of Atlantic Forest, belonging to the Universidade do Vale do Paraiba located, respectively, on the Campus of Villa Branca (Municipality of Jacareí) and of Urbanova (Municipality of São José dos Campos), São Paulo State, Brazil. The species is redescribed and illustrated.
\end{abstract}

Keywords: geographical distribution; Atlantic Forest; Membracinae; taxonomy.

\footnotetext{
1 Especialização em Ecologia - Universidade de Mogi das Cruzes - UMC e Universidade de São Paulo - USP. Docente da Universidade do Vale do Paraíba - Univap. E-mail: lencioni@univap.br.

2 Doutor em Ciências Biológicas (Entomologia) - Universidade Federal do Paraná - UFPR e Docente do Departamento de Zoologia da UFPR. E-mail: amsakakibara@pq.cnpq.br.

Revista Univap - revista.univap.br

São José dos Campos-SP-Brasil, v. 20, n. 35, jul.2014. ISSN 2237-1753
} 


\section{INTRODUÇÃO}

O gênero Enchenopa foi descrito por Amyot e Serville (1843), e, segundo McKamey (1998), possui 24 espécies válidas das quais 15 são do Brasil. Recentemente (ROTHÉA; CREÃO-DUARTE, 2007; STRÜMPEL; STRÜMPEL, 2007; SAKAKIBARA; MARQUES, 2007; 2010), foram acrescentadas, ao todo, mais 6 espécies para o Brasil. As espécies desse gênero são caracterizadas pela presença de uma carena de cada lado do pronoto que vai do ápice do processo anterior até quase às margens laterais, na altura da primeira metade das tégminas; apresentam, também, algumas carênulas oblíquas de cada lado do metopídio. O processo anterior pode ser bastante desenvolvido, alongado, em forma de espada com os dois bordos cortantes ou, então, praticamente ausente, formando apenas uma projeção angulosa acima do metopídio. A coloração é variável, desde pardacenta a preta com manchas brancas ou amareladas. As estruturas das genitálias, sendo muito semelhantes, não servem como caráter diferenciador.

Enchenopa beebei não possui o processo pronotal anterior desenvolvido, tendo sido, por isso, colocada por Haviland no gênero Leioscyta Fowler, quando a descreveu do material coletado na Guiana. Recentemente, Dietrich e McKamey (1995), transferiram-na para o gênero Enchenopa A. \&S.

O interessante é que essa espécie foi coletada no Brasil (São Paulo) somente agora, depois de mais de 80 anos, bem distante da sua localidade-tipo (Guiana). Isso demonstra que a sua população tem uma distribuição geográfica bastante ampla e que, por outro lado, evidencia a falta de coletas de membracídeos, de um modo geral, ao longo da Mata Atlântica.

O objetivo desse trabalho é incluir, para a fauna brasileira, mais essa espécie de Membracidae, cujos exemplares foram coletados no Estado de São Paulo, em pequenas áreas remanescentes da Mata Atlântica (Figura 1), localizadas em Jacareí e São José dos Campos, onde a Universidade do Vale do Paraíba mantém dois Campi, o de Vila Branca e de Urbanova (Figuras 2 e 3).

\section{Enchenopa beebei (HAVILAND, 1925).}

Figuras 4 a 9 .

Leioscyta beebei Haviland, 1925: 239 (loc.-tipo: GUIANA, Bartica); Metcalf \& Wade, 1965: 1315 (cat.).

Enchenopa beebei; Dietrich \& McKamey, 1995: 14 (n. comb.); McKamey, 1998: 201 (cat.)

Descrição original: "Head ochreus, shaded with dark brown, margins of genae arcuate and acutely lobes below; clypeus lobed at its articulation with genae, and with the free margin foliaceus and broadly rounded; eyes grey; ocelli yellow, equidistant between the eyes and median line, and situated just above the level of the centres of the eyes.

Pronotum testaceous yellow, punctured densely in front and more coarsely behind, obtusely angular, not horned at the frontal apex; metopidium high, perpendicular, furnished with a carina which 
is strong and percurrent on the dorsum; humeral angles not prominent; a strong lateral carina on either side extending from the metopidium to the pronotal margin half-way to the apex of the posterior process; three, faintly marked ridges on either side of the metopidium, below and in front of the lateral carina; posterior process acuminate, not quite reaching tip of abdomen, white, black at the apex, and furnished with a bright, chestnut spot on the dorsum between the lateral carinae.

Tegmina chocolate, with a yellow, sub-hyaline patch at the apex. Abdomen, underparts and legs ferruginous black; tarsi yellow.

Long. $5.0 \mathrm{~mm}$. Lat. $2.0 \mathrm{~mm}$. Tegmen. $4.25 \mathrm{~mm}$.

Type: Female. Sexes alike.

A male and female collected at the Station $29-X-20 . . . .$.

\section{REDESCRIÇÃO}

Diagnose.

Pronoto sem processo anterior desenvolvido, de cor de marfim, com uma mancha dorsal castanho-escura, entre as carenas laterais e interrompida na altura do terço final; ápice preto. Tégminas enegrecidas, com uma área translúcida e amarelada no bordo posterior.

Medidas ( $\mathrm{mm})$.

Fêmea/macho: comprimento total 6,17/5,14; comprimento do pronoto 4,93/3,94; comprimento das tégminas 4,18/4,03; comprimento da cabeça 1,18/1,02; largura da cabeça 1,94/1,72; comprimento da cabeça 1,18/1,02; distância entre os ângulos umerais 2,30/1,93.

Fêmea.

Coloração geral marfim, contrastando com a cor negra do restante do corpo. Cabeça marfim, gradualmente enegrecida para o pós-clípeo. Pronoto basicamente marfim, exceto dorsalmente, entre as carenas laterais, logo após o metopídio até, aproximadamente, à metade do processo posterior, castanho-escuro; extremidade negra. Tórax e abdome negros; pernas negras com os tarsos amarelados; tégminas negras, opacas, com mancha amarelo-translúcida no bordo posterior. Vista frontalmente, as marcas de inserção dos músculos de cor ocre-clara em formato de um Y e uma pequena mancha da mesma cor no topo do metopídio sobre a carena média.

Macho.

Semelhante à fêmea, apenas menor.

Revista Univap - revista.univap.br

São José dos Campos-SP-Brasil, v. 20, n. 35, jul.2014. ISSN 2237-1753 
Material examinado.

1 fêmea: BRASIL. São Paulo: Jacareí, Vale do Paraíba / Campus Villa Branca, 30/X/2008/ Col. F. Lencioni; 2312' 33"S/4556'92" W, altitude 559m. 1 macho: Ibidem: São José dos Campos, Vale do Paraíba / Campus Urbanova, 05/IV/2011/ Col. F. Lencioni; 23 ¹6'10”S/4556'23” W, altitude $613 \mathrm{~m}$. Depositado na Coleção de Entomolgia Pe. J.S. Moure, Departamento de Zoologia, UFPR, Curitiba, Paraná (DZUP).

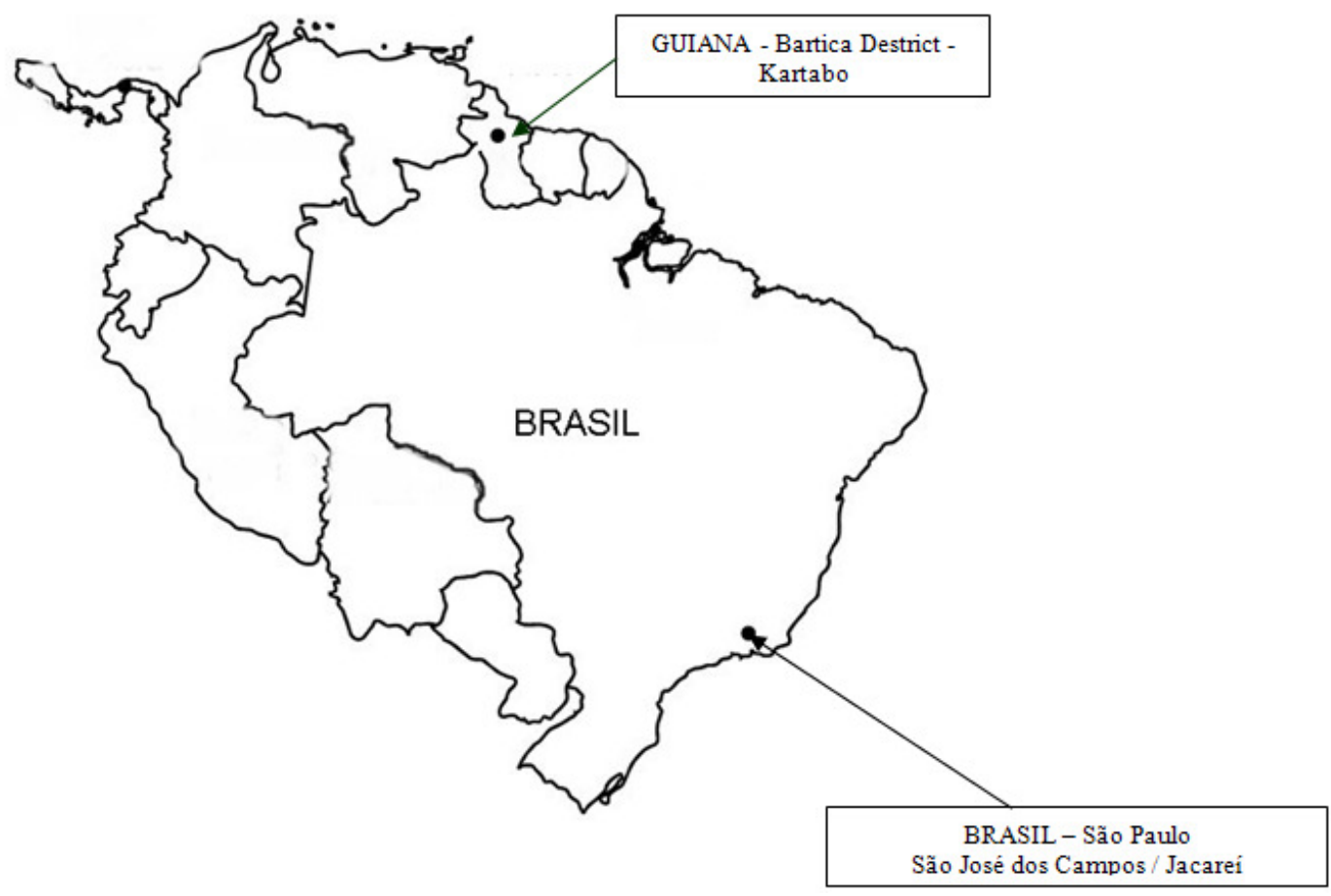

Figura 1 - Mapa da localização das coletas. 


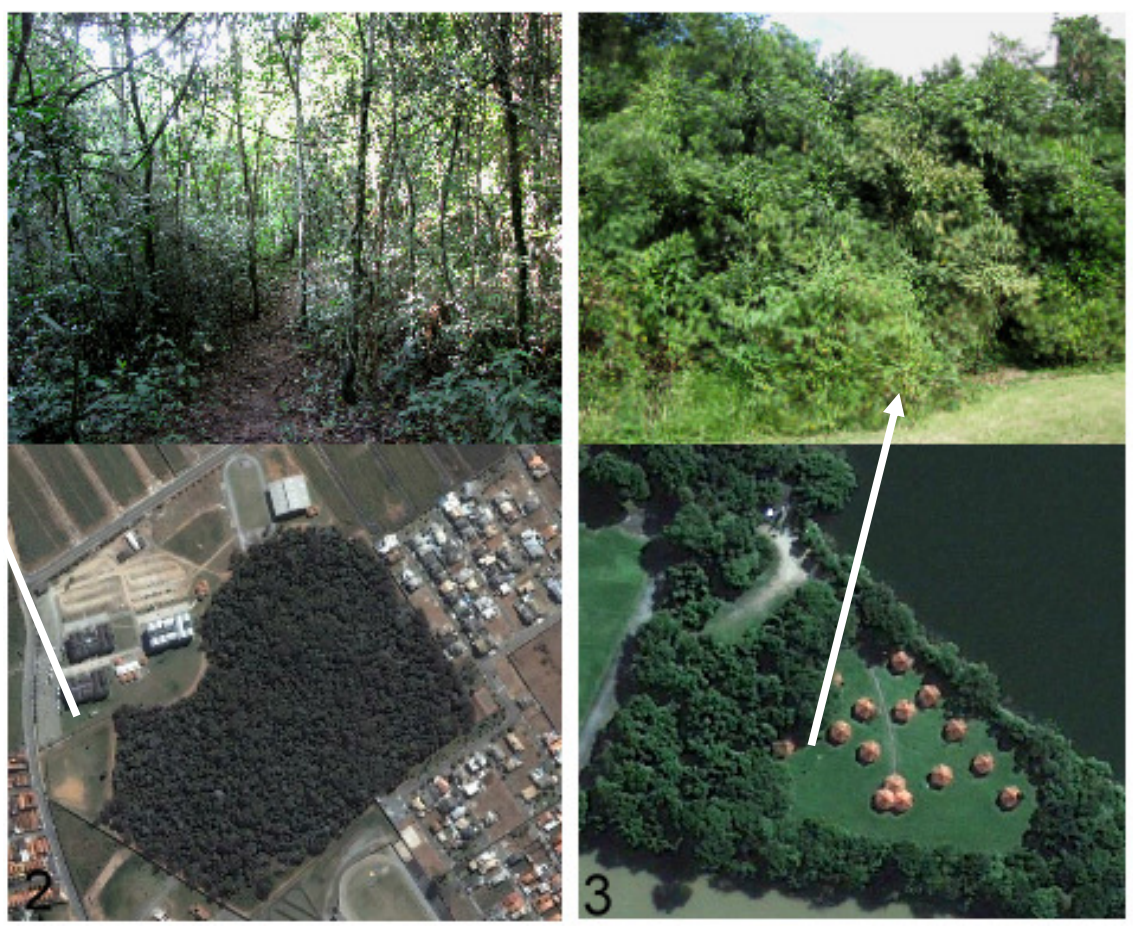

Figuras 2 e 3 - Aspectos das áreas e locais das coletas de Enchenopa beebei. 2. Villa Branca (Jacareí); 3. Urbanova (São José dos Campos).

\section{Comentários.}

Enchenopa beebei é uma espécie facilmente identificável pela sua coloração, diferente da de todas as outras do gênero. O contraste entre o marfim do pronoto e o negro das tégminas a faz ímpar entre as espécies descritas até o momento.

Os locais onde foram coletados os exemplares são remanescentes da Mata Atlântica. De hábitos solitários, os indivíduos foram observados pousados sobre a folhagem, na borda da mata. A mata do Campus Urbanova é uma faixa estreita de mata ciliar do Rio Paraíba do Sul, local bastante ensolarado e com inúmeras plantas da família Solanaceae (Solanum sp. Alchornea sp.) e, também, Piperaceae. A área do Campus Villa Branca é muito semelhante quanto à vegetação, mas muito seca e distante de algum curso d'água. Infelizmente, não foi possível determinar a planta hospedeira. 


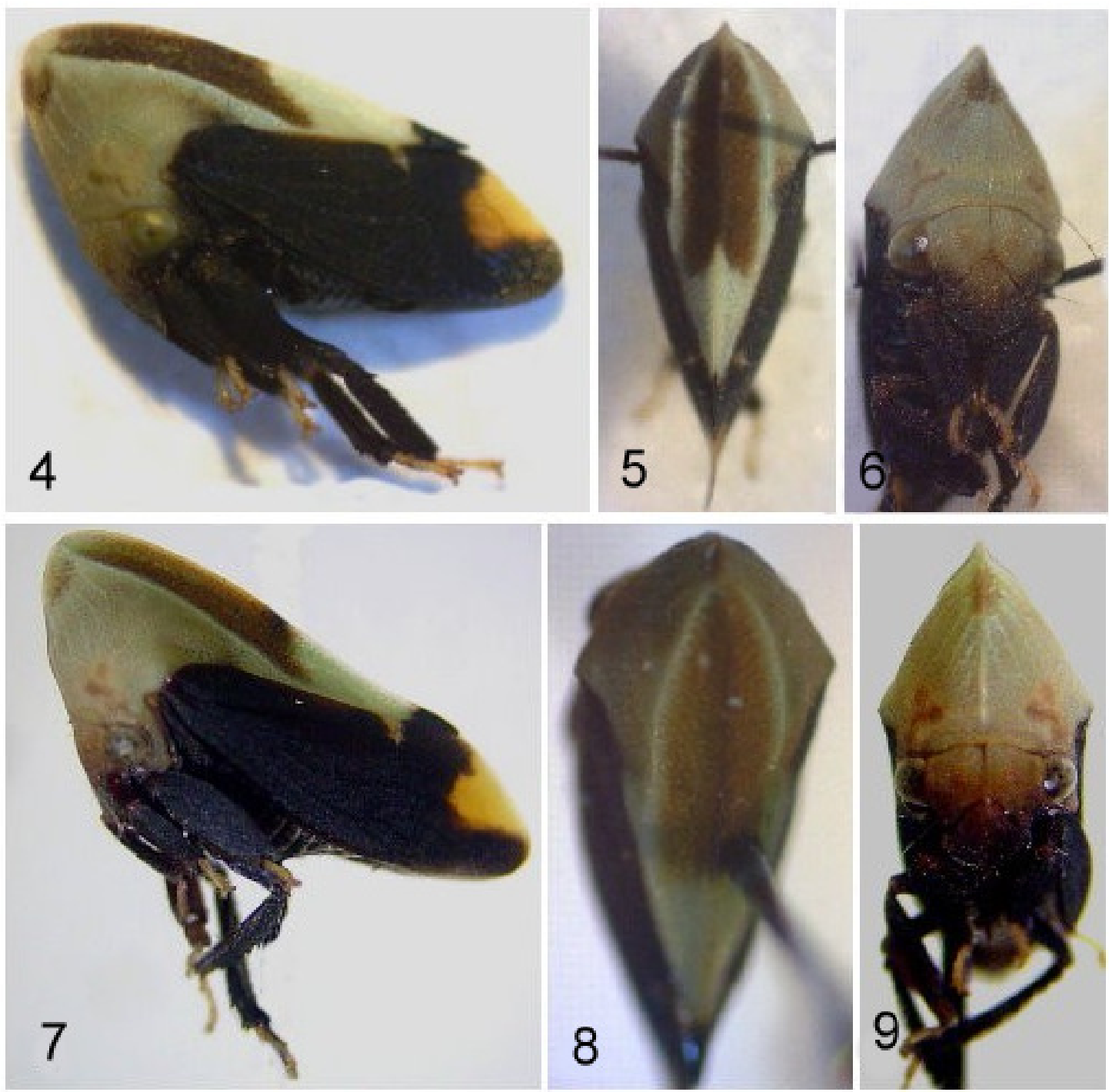

Figuras 4 a 9 - Enchenopa beebei: 4-6. macho; 7-9. fêmea; 5 e 8. vista dorsal; 6 e 9. vista frontal.

\section{REFERÊNCIAS}

AMYOT, C. J. B.; SERVILLE, J. G. A. Histoire Naturelle des Insectes: Hémiptères. Deuxième partie. Homoptères. Homoptera Latr. Paris, France: Librairie Encyclopédique de Roret, 1843. p. 455-676.

DIETRICH, C.H.; MCKAMEY, S.H. Two new Neotropical treehopper genera and investigation of the phylogeny of the subfamily Membracinae (Homoptera: Membracidae). Proceedings of the Entomological Society of Washington, v. 97, n. 1, p. 1-16, 1995.

FOWLER, W. W. Order Rhynchota. Suborder Hemiptera-Homoptera. Biologia CentraliAmericana, v. 2, p. 1-24, PL.1-2. 1894.

HAVILAND, D. M. The Membracidae of Kartabo, Bartica District, British Guiana, with descriptions of new species and bionomical notes. Zoologica, v. 6, n. 3, p. 229-290, 1925. 
MCKAMEY, S. H. Taxonomic Catalogue of the Membracoidea (Exclusive of Leafhoppers). Second Supplement to Fascicle 1-Membracidae of General Catalogue of Hemiptera. Memoirs of the American Entomological Institute, v. 60, p. 1-377, 1998.

METCALF, Z. P.; WADE, V. General Catalogue of the Homoptera. Asupplement to fascicle IMembracidae of the General Catalogue of the Hemiptera. Membracoidea. Sec. II. Raleigh, North Carolina State: University at Raleigh, 1965. p. 745-1552.

ROTHÉA, R. R. A. D.; CREÃO-DUARTE, A. J. Duas novas espécies de Enchenopa Amyot \& Serville (Hemiptera, Membracidae) do semi-árido do Estado da Paraíba, Brasil. Revista Brasileira de Entomologia, v. 51, n. 1, pp 35-37, 2007.

SAKAKIBARA, A. M.; MARQUES, O. M. Espécies novas de Membracidae (Hemiptera, Cicadomorpha) coletadas em um agroecossistema cacaueiro da região sul da Bahia, Brasil. Magistra, v. 19, n. 1, pp. 82-87, 2007.

SAKAKIBARA, A. M.; MARQUES, O. M. Uma nova espécie de membracídeo (Hemiptera, Membracidae) coletada em plantas da família Loranthaceae. Magistra, v. 22, n. 2, pp. 134-136. 2010.

STRÜMPEL, H.; STRÜMPEL, R. Eine neue Art der Gattung Enchenopa Amyot \& Serville, 1843 (Hemiptera: Membracidae: Membracinae) aus Brasilien. Entomologische Mitteilungen aus dem Zoologischen Museum Hamburg, v. 14, n. 177, p. 421-427, 2007. 